\title{
The (non) use of prioritisation protocols by surgeons Kevin Dew ${ }^{1}$, Maria Stubbe ${ }^{2}$, Lindsay Macdonald ${ }^{2}$, Anthony Dowell ${ }^{2}$ and Elizabeth Plumridge ${ }^{2}$
}

\author{
${ }^{1}$ Sociology Programme, Victoria University of Wellington, New Zealand \\ ${ }^{2}$ Department of Primary Health Care and General Practice, University of \\ Otago, Wellington, New Zealand
}

\begin{abstract}
Priority setting and rationing is a dominant feature of contemporary health policy. In New Zealand, clinical priority assessment criteria (CPAC) tools have been developed to make access to elective surgery more equitable and efficient. Research was undertaken to identify how surgeons used these tools in the consultation. Forty-seven consultations with 15 different surgeons have to date been video- and audio-recorded. There were no instances where CPAC tools were explicitly used in the consultation. Drawing on the methodology of conversation analysis and the concept of news delivery as developed by Maynard, this paper argues that the delivery of diagnoses and treatment plans can usefully be seen in part as the delivery of bad or good news. Using three case studies to illustrate the argument, it is suggested that the interactional work required in the delivery of such news challenges the ability of clinicians to use protocols such as CPAC. The analysis sheds light on important consultation processes that need to be more carefully considered when designing interventions to influence clinician behaviour. In order to influence the behaviour of clinicians to achieve policy goals, greater attention needs to be paid to the interactional demands of the consultation process.
\end{abstract}

Keywords: elective surgery, prioritisation tools, doctor-patient interaction

\section{Introduction}

Dominant discourses in contemporary health policy are those of priority setting and rationing (Joyce 2001). Nation-states have pursued a number of paths in attempting to achieve rationing. These include: limits being placed on what treatments can be provided, as with the Oregon Medicaid Initiative (Birkmeyer and Welch 1993); tools developed to manage waiting lists, as with the Western Canada Waiting List Project (Taylor and Hadorn 2002); technology appraisals used to recommend treatments, as with the National Institute for Health and Clinical Excellence in Britain (Steinbrook 2008). Considerable effort has been made in New Zealand since the late 1990s to move away from a system of implicit rationing, where discretionary decisions were made within fixed budgets, to a system of explicit rationing, where the rules of allocation are more transparent (Dew et al. 2005). An elective services project was established that aimed to define criteria for prioritisation such as need and ability to benefit, to make rationing transparent and to promote national consistency. 


\section{Kevin Dew et al.}

This policy development was in response to concerns about the length of waiting lists and declining public confidence in the system.

As a result of this new policy, clinical decision-making tools were introduced into the public hospital system. These were developed in different forms for the different specialties with high levels of input from clinicians with the aim of making access to publicly funded elective surgery equitable and efficient (McLeod et al. 2004). These decision-making tools, known as Clinical Prioritisation Assessment Criteria (CPAC), are used in conjunction with financial thresholds. If a CPAC score is above the financial threshold of the relevant hospital then treatment can be offered.

The presumption was that these tools would alter referral processes appropriately to ensure that clinical priorities were the basis of referral decisions rather than any other factors like patient or surgeon lobbying, language difficulty, or cultural difference between patient and surgeon. Berg (1997) argues that terms like guidelines, protocols and practice policies have been used to describe the same thing, arguing that 'they can be read as a set of instructions telling medical personnel to do a certain thing in a certain situation' (1997: 2). These protocols relate to efforts to standardise medicine, and as such define medical practice as the logical and sequential application of science.

An evaluation of the elective services project, however, reported that surgeons claim to use the tools in a variety of ways - from deploying them as strict protocols, to filling them out once a decision had already been made (Dew et al. 2005). This research, based on interview data, suggested that 'Much of the rationing is still implicit in that there is still a reliance on the discretion of clinicians to weight subjective criteria' (Dew et al. 2005: 10). There has been other research exploring what health personnel think about clinical protocols (Flynn and Sinclair 2005) and raising issues about the integrity and credibility of clinical protocols (Genuis 2005).

The research reported upon in this paper attempts to explore the issue of how clinical protocols do or do not fit into the actual interactional nature of the surgeon-patient consultation. The original research question was to see how naturally occurring data could provide insight into the ways in which clinicians weighed up objective and subjective criteria in the tools when making decisions to refer patients to elective surgery. Many of the tools have subjective criteria, such as the impact a condition has on social functioning, as well as objective criteria such as absence of active infection. For example, the orthopaedic CPAC clinical score is based on degree of disability, level of pain, potential for harm through delay, social circumstances and ability to work (Ministry of Health 2007).

\section{Data collection}

The data base that has developed out of this study, as of February 2009, included 228 videoand audio-taped consultations between health practitioners and patients, with 47 of those consultations being between surgeons and patients. As surgeons are required to use CPAC tools to book patients in for elective surgery their consultations are the focus of this paper. Ethical approval for the study was obtained from the Wellington Ethics Committee.

Surgeons were approached and asked to consent to the study. Fifteen surgeons consented from a variety of specialties including general, breast, vascular, orthopaedic, cardiothoracic, ear nose and throat and neurosurgery as well as ophthamology. On agreed days a researcher would attend the clinic and obtain consent from patients who fitted the inclusion criteria. Patients were excluded where conditions were acute and if they were under 18 years of age. Those who gave verbal consent were then asked to read and sign a consent form, and were 
assured that they could change their mind at any stage and ask for all or part of their recording to be deleted.

The length of consultations ranged from four minutes (routine follow-up) to 41 minutes. These consultations resulted in a range of decisions including six decisions not to refer for further tests or treatment, 13 decisions to refer for diagnostic tests, 13 decisions to operate and nine decisions to make follow-up appointments (more than one decision could be made in any one consultation).

\section{Methodological approach}

Conversation analysis was seen as an appropriate tool to shed light on how clinical decision making relates to explicit rationing because it focuses on the sequence and unfolding of talk as it occurs in social interaction, and because conversation analytic approaches do not rely on the cognitions, rationales and justifications of those involved in the social interaction, but look rather to the rules of interaction itself.

Conversation analysis is a common methodological approach in research on healthcare interactions, including research looking at encounters between patients undertaking chemotherapy and their doctors (Diaz 2000), consultations related to antibiotic medication (Heritage and Stivers 1999), and psychiatric consultations (Seale et al. 2007). It allows 'rules of interaction' to be identified, which in turn enhances our understanding of the outcomes of social interaction. The term 'rules' here does not refer to a rigid set of prescriptive norms, but rather, is used in the sense of 'standards that determine what constitutes an activity of that kind, whether the activity has been performed correctly and so on' (Hutchinson et al. 2008: 41). An initial issue for researchers is then to describe the 'activity', and the role of the researcher is to pay close attention to the details of social interaction in order to generate an understanding of the ordered nature of the interaction (Heritage and Maynard 2006).

At the completion of the data collection, it was discovered that for this sample none of the surgeons explicitly used or referred to the applicable CPAC tools in the consultation. CPAC tools had to be completed as this documentation is required in order for a patient to access elective surgery, but the surgeons were presumably doing this outside the consultation itself or delegating the task to other clinic team members. Previous research on the use of CPAC found that surgeons and registrars were told by hospital administrators to use the CPAC tools even though they were not necessarily told how to use them (McLeod et al. 2004).

This outcome was not anticipated for three reasons. First, the policy intention was that CPAC tools would aid decision making and as such would be used in the consultation as decisions were made (Dew et al. 2005). Secondly, this finding was in marked contrast to a training video for medical students which showed a surgeon explicitly using the tool in the consultation. This involved the surgeon working through a structured questionnaire where a pre-determined list of questions is asked of the patient and the patient's answer is matched with a category (such as mild pain, severe pain, very severe pain) that carries a particular score. Thirdly, it was expected that the CPAC tools might in fact be used by surgeons to deal with interactional difficulties, as in a study by Rhodes et al. (2006) where nurses used checklist tools as a resource for dealing with issues such as not knowing how to respond to patient concerns; checklists allowed them to move on to the next item. We hypothesised that the CPAC tools might be used, for example, when treatment outcomes did not conform to patient expectations. Any discussion could then potentially be circumvented by referring to the tool score as an 'objective' means of determining treatment plans. 


\section{Kevin Dew et al.}

Given these points, the question arose as to why surgeons were not using these tools as part of the consultation interaction. So the research question shifted from trying to understand how surgeons used the tools within consultations, to understanding what was occurring in the interaction that might preclude the use of such tools. To answer this question, we therefore need to understand what activities were being performed, and whether or not following a protocol like CPAC might mean that this activity would not be performed 'correctly'. In other words, are the rules that allow participants to identify the activity as being 'a consultation' in conflict with the interactional requirements of administering a protocol that determines whether someone can access a rationed health service like elective surgery?

Maynard's (2003) research on the delivery of good news and bad news provided insights into the consultation when the issue of diagnosis was conceptualised by one or both participants as either bad news or good news. Maynard makes a number of suggestions for how the telling of bad news typically unfolds during an interaction. He argues that 'The quality of a news presentation is inseparable from the relationship between deliverer and recipient...Forecasting the news through demeanour, talk, and gestures provides for mutuality between deliverer and recipient and enhances the likelihood of recipients comprehending the news' (Maynard 2003: 249). Clinicians typically employ the format of the 'news delivery sequence' (2003) of 'everyday' conversation. The format consists of a 'pre-announcement' or inquiry followed by the 'announcement' of the news/diagnosis, which is in some way 'received' by the patient. This is then often followed by some 'elaboration' of the news/diagnosis by the clinician and then some 'assessment' is displayed by the patient as they realise its import (Maynard and Frankel 2006). Maynard has shown that clinicians typically first ask questions that encourage patients to display their expectations, known as a 'perspective display series'. Patient expectations are then 'co-implicated' to secure patient alignment with the diagnosis. If interactional 'work' like this is not done, patients may misapprehend, not align or even oppose or reject diagnostic 'news'.

It is argued in this paper that the use of standardised tools to determine decisions would disrupt the interactionally important news delivery sequence. In so doing, they would breach the socially sanctioned world of everyday life, and we argue that this is a likely explanation for the observed non-use of the CPAC tool within the 47 consultations collected for this study. Following Garfinkel, Maynard argues that 'Morality... refers to ways that members of society 'trust' the world of everyday life through adherence to a set of reciprocally binding assumptions' (Maynard 2003: 200). These 'binding assumptions', for Maynard, include the ways in which good and bad news is delivered. Externally-derived rule-bound protocols such as CPAC are likely to challenge the interactional achievements required in the delivery of clinical news.

On the basis of the data analysed as part of this study it is argued here that the delivery of a diagnosis - whether good or bad - is akin to the delivery of news - whether good or bad, and that checklist tools, if used, would hinder the socially appropriate delivery of this news. In a consultation about elective surgery, the relevant activity is to determine whether someone is willing to and should undergo surgery, not to prioritise. To combine these different activities into the one consultation runs the risk of disorganising its interactional structure. In addition, a high level of interactional competence is demanded of surgeons in order to deliver a diagnosis and negotiate a treatment plan successfully. The complexity of this interactional achievement is at odds with the fixed, agenda-like interaction required by protocols such as the CPAC tools. Specifically, the issues of diagnosis and treatment plan are interwoven in the interaction, as any news delivery requires elaboration and an exit. The natural exit point in relation to diagnosis is what is to be done about it, if anything can be done. We argue here that the use of CPAC tools would actually hinder this naturally occurring sequential organisation, as they could not be inserted into the diagnosis sequence 
without being interactionally disruptive, and that this explains their absence in the consultations we recorded.

We illustrate next why this is so by examining in detail three surgical consultations from our data set. In the first example, very bad news is successfully delivered with careful interactional work, such that the patient 'realises' but is not overwhelmed by its import. The second example, by contrast, involves the delivery of apparently 'good' clinical news. There is no evidence in either of these interactions of a CPAC protocol being used, although a need to complete 'the paperwork' is explicitly referred to by the surgeon at the end of the second example. The third case provides a point of comparison: this is a composite consultation where an ophthalmology registrar examines a patient and plans surgery which is then immediately followed by a nurse consultation where the CPAC score sheet is filled out. This is the only example in our data set where we have interactional evidence of a health professional explicitly orienting to and implementing the relevant CPAC protocol.

\section{Analysis}

In this first illustrative case Patient A is happy in her belief that she now experiences good health. Although this particular consultation would probably qualify as an 'override' in CPAC terms (i.e. one where the CPAC tool would not have to be filled out as there is a concern for cancer here) the consultation provides very useful insight into the complex interactional work required to obtain alignment between the surgeon's proposal and the patient's understanding. In particular, it makes clear how the explicit use of decision aids in such a context would interfere with the delicate interactional work the surgeon undertakes as he changes the patient's optimistic belief and delivers bad news, but tempered with good news projections in that something can be done about the situation. The surgeon (SG) begins with an inquiry designed to elicit a perspective display series from the patient (PT):

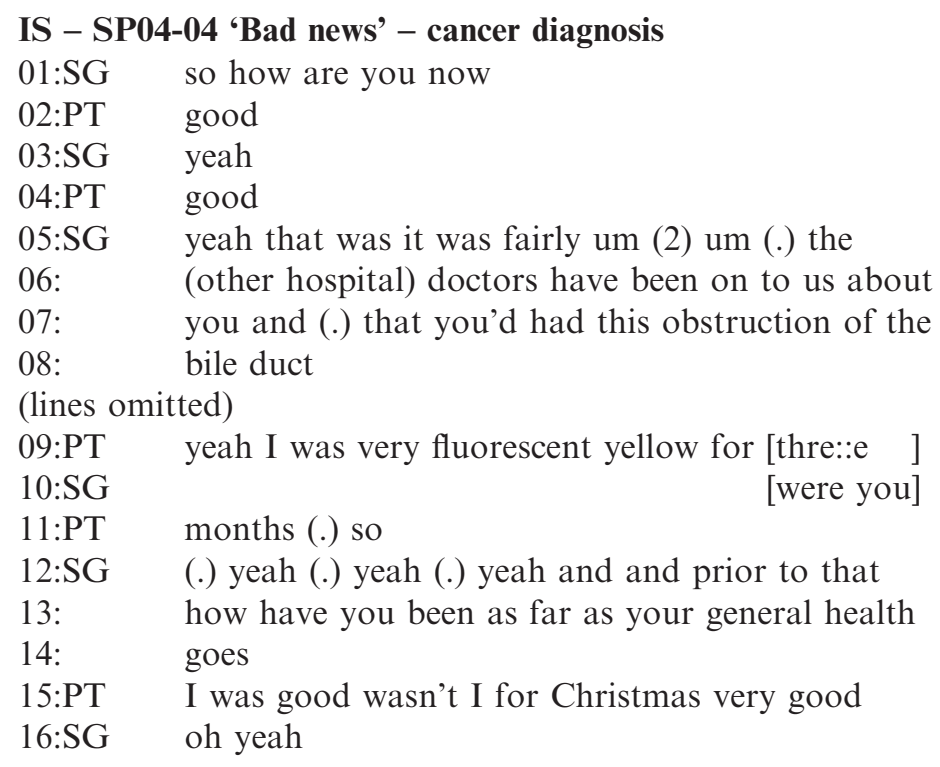

This manifests a repeating pattern in this consultation. The surgeon asks about the patient's health and receives invariably positive responses. To these he makes a non-committal 
response token like 'yeah' (lines 3, 5, 12 and 16) and an elaboration which in some way queries her assessment or projects some import more serious than her response recognises. In total the surgeon queries the patient's self-assessment nine times. She maintains 'no problem' responses no matter how he builds his questions to make this a dispreferred response (Maynard 1992). ${ }^{1}$ That is, the questions are designed to elicit a problem - the preferred response - but instead elicit the dispreferred 'no problem' response. For example:

17:SG in the normal sequence of things (.) are you

18:

19:PT

20:SG reasonably active or (.)

$$
\begin{aligned}
& \text { very [active ] } \\
& \text { [active yeah] }
\end{aligned}
$$

The question asked at lines $17^{2}$ and 18 prefer a downgraded response - reasonably active, or something less than that. But instead the patient upgrades the response to 'very active'.

The surgeon breaks this cycle finally by asking if doctors had explained that a blockage such as hers in the bile duct is 'normally' a growth:

21:SG did they explain that?

22:PT yes

23:SG yeah

24:PT but they also said that there was no trace of (.)

25: cancer in the (.)specimen that they [took]

26:SG

27:

28:

29:

30:

31:

32:PT

[y-yes] well 33:SG conclusive and I think we'd- you know we'd (.)

34:

35:

36:

37:PT

(...) which isn't unusual (.) you know it's just um

(.) they (.) they can- they just draw out some cells from the bile duct itself (though) you'd norm-you'd normally get (.) (should) get an answer on that so the-the-fact that () isn't you knowconclusive usually have to (.) a-assume that a blockage of the bile duct in that position is um through a malignancy (.) $[\mathrm{mm}]$ [oh ]

The patient recognises that the surgeon has now foreshadowed some very bad 'news', although her first response on lines 24-25 is to again assert a no-problem reading: 'but they also said that there was no trace of (.) cancer'. The surgeon then delicately elaborates in such a way that the patient herself pre-emptively ends his sentence on line 32 with its fatal import: past tests are not 'conclusive'. This leaves the surgeon able to repeat the patient's ending and elaborate until she finally 'realises' what is being discussed. Her 'oh' on line 37 is a news receipt, indicating a moment of recognition and 'change-of-state' (Heritage 1984). Such news receipts are not a regular response to medical assessments, but do occur when the doctor's assessment contrasts with the patient's version (Heath 1992). The surgeon then talks of an operation:

38:SG so (.) um if we were (.) if we were going to take 39: $\quad$ this further then the next step would be just to do 40: $\quad$ to do a small operation (.) to look um (.) in your 
41: $\quad$ abdomen through some small holes and (.) some

42: $\quad$ instruments just to try and get more information er

43: $\quad$ as to whether it's localised ah or not

There is then some discussion of whether hers could still be a no-problem condition and a lengthy elaboration of evidence to the contrary before the patient herself prescribes the action of further investigation that has been in numerous ways tacitly canvassed:

44:PT could we go ahead and [do that mister () and ]

45:SG [y-yeah okay yeah yeah] an-

46: $\quad$ an-and then after that we'll know y-you know a bit

47: more.

The patient has now not only 'realised' the diagnostic news but assessed it as needing to be followed up. The diagnosis, unexpected as it was, has been delivered by the surgeon with such competent attention to the patient's orientation that she is highly aligned with the surgeon by the end of the process.

This first example illustrates the point that while diagnostic news of a serious condition is unlikely to be welcome, patients may be made more ready or less ready to realise and cope with it. Where such consequential matters as surgery and personal health are involved, a very high level of interactional competence is required. Patient perspectives must be gauged; a diagnosis must be formed and delivered appropriately; a treatment regime has to be discussed and worked out. In such circumstances 'best practice' is not to be derived from tightly scripted clinical decision-making tools.

In this second case the surgeon provides a 'diagnosis' and treatment plan that is oriented to as good news by both the surgeon and patient. Patient B presents with subjective symptoms of periodic intense pain of the abdomen. The surgeon opens the consultation with reference to his colleague diagnosing a 'volvulus or twisting of the bowel'. The surgeon then inquires into the patient's current situation with 'could you just tell me a little bit about yourself', and then later delves further into the history of the problem with 'could you just tell me a little bit about (.) what what you've noticed with the bowels and so on'. At the completion of the patient's account the surgeon describes the problem to the patient as follows:

\section{IS - SP03-02 'Good news' - surgery offered to correct volvulus.}

01:SG it's basically like a (.) the large bowel's like a

02: $\quad$ there's a sort like of like an inverted u shape

03: $\quad$ tube but $=$

04:PT $\quad=$ yeah $=$

05:SG = in some people it it's sort of got (.) part of

06: $\quad$ it's very elongated (.) and it's on quite a narrow

07: (pedicle) and that allows the whole thing to twist

08: $\quad$ around $=$

09:PT = yeah $=$

10:SG $=$ so it blocks off when it twists it (.) the tube

11: gets blocked off at two points and they sort of ()

12: (close) system (.) er and that's obviously when

13: you're getting the pain and so on $=$ 
14:PT $\quad={ }^{\circ}$ yep $^{\circ}$

15:SG yeah now (.) in in some people (.) particularly in

16: people er you know er like yourself who have had it

17: from an early age it the they have a (.) a

18: motility problem with the bowel generally in other

19: words (.) although (2) it's the sigmoid colon which

20: is the commonest place for this to happen the whole

21: bowel may be a little bit sluggish (.) [you know]

23:SG er and (.) what we what we generally when if it

27:

28:

29: looks like it's just the sigmoid colon that's causing the problem particularly in a in a young person like yourself we would just deal with that bit that's twisting (.) um but it may be (.) that further down the track er y- you might have (.) on going problems with [constipation] [yeah ye- ] you know if we're talking about surgery we'd be (.)

36:SG that's twisting it and (I'll just straight) take

37:

38:PT

39:SG talking about doing a limited op-operation to deal with the bowel's causing the trouble yeah

In this transcript we can see the surgeon moving from a description of the problem to a possible treatment plan for the problem. Throughout, the patient provides positive response tokens with a series of 'yeah's and 'yeps' at lines 4, 9, 14, 22, 30, 35 and 38. The surgeon's presentation of his assessment of the problem and how the problem could be remedied does not clearly elicit the patient's expectations, but nevertheless, throughout the consultation the patient aligns in an increasingly positive manner to the surgeon's suggestions.

Although there has been no actual agreement for an operation to be undertaken the surgeon continues the discussion about aspects of the operation stating that:

40:SG (you know) in a young person like yourself we'll I

41: think one would deal with the (.) the the problems

42: $\quad$ it's really (.) you know [causing] this twisting

43:PT [(yeah) ]

In line 40 the phrase 'in a young person like yourself' is repeated from lines 25-6, building up the case for the surgical intervention and on line 41 use of the impersonal pronoun 'one' provides an abstract authority for the approach to the problem. The surgeon then examines the patient and after this provides more detail on the proposed operation:

44:SG when you've got this condition with this volvulus

45: $\quad$ the bowel's very floppy and easily accessible so we 
46: $\quad$ can do this through a very small hole okay

47:PT oh yeah [absolutely yeah]

48:SG [right ]

Again we can note on line 47 the patient's very strong agreement 'oh yeah absolutely yeah' to the surgeon's concluding words of 'okay' and 'right'. The surgeon then outlines possible risks prefaced with 'it is a fairly straightforward operation' and likely post-operation scenarios. After agreeing on a time for the operation to occur the surgeon states:

49:SG yep okay (.) um ((tut)) (.) right so all I need to

50: $\quad$ do then just get the paperwork organised and er (.)

51: we'll we'll try and work to- towards those sort of

52: dates I think that's a reas- I sort of think it

53: $\quad$ needs to be done (.) you know because it's it's

54: $\quad$ going to happen again (.)

55: [and er there's no there's no] way of predicting =

56:PT [yeah yeah that's all right ]

57:SG = when or where or

58:PT yeah

Then later again the surgeon states:

59:SG all right so that's okay we'll we'll get the

60: paperwork organised okay

61:PT excellent

This surgeon offers over five minutes of almost uninterrupted monologue and then a great deal of re-iteration in relation to the surgery. Note that lines 50 and 59-60 refer to a postconsultation activity of 'paperwork', which would include assigning a CPAC score so that the patient can have access to publicly funded elective surgery. At no stage during the consultation, however, is any scoring undertaken explicitly. Neither does the surgeon at any stage provide an explicit opportunity for the patient to query the course of action, but there is also no signal from the patient that he is uncomfortable with the course of action. On the contrary, throughout the consultation the patient produces increasingly strong forms of affiliation with the surgeon's proposal, seen again in lines 56 and 58 and culminating in a response of 'excellent' on line 61. The 'agreement' to undertake surgery therefore is interactionally achieved, with the parties to the interaction co-operating to achieve the agreed outcome.

This example of a 'good news' consultation, where there is an apparent alignment between the surgeon's proposal to perform surgery and the patient's understanding of his condition and acceptance of the proposed treatment, illustrates the important interactional work undertaken by the surgeon, even in what appears to be a 'straightforward' case (in terms of clinical decision making). At no time during the entire consultation does the surgeon ask a direct question of the patient - do you want surgery or not? Rather, he responds to the response tokens and affiliations of the patient. The surgeon provides a sequence of talk that suggests that he is working towards an already pre-determined goal or recommendation, namely, to undertake the surgery, and through his inquiries into the patient's current social arrangements and experience of the condition, he discerns the patient's positive alignment to the proposal. 
This alignment is built up incrementally, not explicitly in a linear or additive fashion but interactionally, with both participants responding to each other to co-construct and affirm the decided outcome. The predetermined, scripted form of the CPAC tools where scores are added up on the basis of an invariant set of questions would not allow for such an interactional achievement. Once an agreement has been reached and the date of the operation has been set the surgeon can then attend to the 'paper work'. The use of a decision-making protocol such as the CPAC tool would clearly be redundant in terms of this interaction due to the implicit presupposition on the part of the surgeon that this surgery is necessary and justifiable. It can also be argued that attending to 'the paperwork' prior to the alignment of proposals and understanding might well jeopardise the occurrence of an alignment between clinician and patient.

As stated, there were no examples in our data set of surgeons using CPAC within the consultation. We do, however, have one example of an ophthalmology registrar examining a patient and planning surgery which is then followed by a nurse filling out a CPAC score sheet. This example allows us to see even more clearly the way in which the activity of prioritising, when separated out explicitly, has its own interactional delicacies - but is also quite different from the activities of diagnosing and planning treatment. At the end of the nurse's scoring the caregiver of the patient is not clear about the outcome, and therefore makes a request to ascertain the 'news' - interactionally a very different process from the news delivery outlined in the above cases.

In this consultation the problem presentation of deteriorating vision is provided, but this time by a caregiver (CR) as the patient has some intellectual impairment. The patient's wife (WF) is also in the consulting room. After the specialist (SP) asks 'what's the problem with your eyes' the caregiver provides an account of the patient (PT) having walking problems and the GP finding that 'he was really blind in one eye' and had 'a cataract in one eye', explaining the reason for the referral. This is followed by the specialist inquiring into specific aspects of the patient's health, medications, living circumstances and activities. The specialist then tests the eyes of the patient. After the tests have been completed:

\section{TS - SP19-01 'Good news' - cataract surgery offered.}

01:SP there are definitely cataracts there and that's the

02: $\quad$ main reason why you can't see so well (1.5)

03:PT Mmmm

(lines omitted)

04:SP uumm the question is whether (.5) you were keen

05: $\quad$ for us to do something about that Barry (.5) would

06: $\quad$ you like us to fix that if $w-$ if $y$

07: $\quad$ if if you:: (.5) er if we can $\uparrow($.$) would you like$

08: $\quad$ us to do that (.)

09:PT oh yes plea[se ]

10:SP [yeah]

11:WF what

12:SP yeah

13:CR said to have have the operation to

14:

15:SP [cos this will invol]ve an an operation to: u:m

16: $\quad$ to replace the um the cloudy lens (in the) eye with

17: an artificial lens and it's quite a safe procedure

18: $\quad$ um and most people get good vision afterwards 
The specialist attempts to ascertain the patient's attitude towards an intervention on lines 0405 'the question is whether (.) you were keen for us to do something about that Barry'. He then reformulates this more clearly as a direct question but with a post-posed if-modifier which indicates that this is not an unconditional offer: 'would you like us to fix that if $w$ - if $y$ - if if you:: (.)' (lines 06-07). The dysfluency and 'word search' here indicates some kind of interactional delicacy, possibly a reluctance on the part of the doctor to make the rationing aspect of this decision explicit by saying something like 'if you (score highly enough)'. This interpretation is supported by the way the doctor eventually reformulates his question with a non-specific conditional clause first, which serves to emphasise more clearly that there are no guarantees, while at the same time avoiding any explicit reference to why it might be that 'we can' (or not) 'fix' the problem: 'er if we can would you like us to do that' (lines 07-08). The patient provides a positive response and the specialist provides some further information about the safety of the procedure on lines 17-18. The caregiver then tries to re-confirm with the patient's wife:

19:CR do you want to have the operation for Barry to have

20:

21:WF yeah

22:CR yeah

23:SP if possible you know

Here, after already establishing that an operation is desired, the specialist re-introduces some doubt (earlier signalled at line 07 by the conditional 'if we can') with the phrase 'if possible you know' on line 23. These doubts refer to the CPAC score and whether or not it will be high enough for the patient to qualify for elective surgery. After other topics such as some other signs of retinal damage have been discussed the following occurs:

24:SP we'll put you on the list

(lines omitted)

25:SP $\quad=$ so I- I guess (.5) the only question left is

26: whether we are keen to do it under local or general

27: $\quad$ have you got any (.) you know feelings about that

So, again the decision has clearly been made. The 'only question left' (line 25) is whether a local or general anaesthetic would be preferred. This is further reinforced when after the discussion about the type of anaesthetic to use the specialist states:

28:SP $\quad$ so I'll just we just need to do some paper work now

The specialist then talks of the need for a pre-assessment before the actual surgery and then states:

29:SP we'll just need you to do some forms with the

30: $\quad$ nurses now just to go through a few questions about

31: $\quad$ um

32:CR $\mathrm{mm}[\mathrm{hm}]$

33:SP [u- u]- u- about about the daily life

34:CR $\mathrm{mm}$

35:SP and um (.) I'm sure that you'll get enough points to

36: $\quad$ get on the waiting list 
This is referring to the CPAC. We do not have data on the interaction that occurs between the specialist and the nurse when the specialist leaves the room and before the nurse comes to deliver the prioritisation form; however, when the nurse (NS) comes in she says hello and then:

37:NS here I am to do the consent er the cataract scoring

The nurse then follows this with:

38:NS okay let's do this quick and then you can get out 39: of here ay

By this time the patient, caregiver and wife have been at the public hospital for several hours. Then:

40:NS ${ }^{\circ}$ what are we up to (.) sixteen isn't it ${ }^{\circ}$ so this is

41: $\quad$ about how you're managing with your eyesight as it

42: $\quad$ is at the moment

The nurse's reference to 'sixteen' (a score that the specialist has already recorded on the assessment form on the basis of the distance vision test he performed) on line 40 is said sotto voce. The nurse then proceeds through the form:

43:NS So do you have trouble seeing people's faces $\uparrow$ and

44: $\quad(($ PT shakes his head $))$

45:NS if someone's walking towards you you know who they

46: $\quad$ are

47:PT ye::s

48:NS yep

49:CR $\mathrm{mm}($.$) depends upon where he's sitting and what's$

50: $\quad$ he doing

The patient is providing 'incorrect' responses, both in terms of his actual vision as assessed by the caregiver, and in terms of the planned outcome - and so the caregiver provides an alternative account to the patient's on line 49. This continues:

51:NS you know when they say what the temperatures are

52: and things like that can you see that on the $\mathrm{t} v \uparrow$

53: $\quad$ (.) or do you have to get quite close (1.5)

54:PT oh ye::s

55:NS yep (.) okay (2.0)

56:CR I think when (.) with

57:NS ye[p ]

58:CR [because ((FIR]ST NAME- WF))'s been practising

59: when he with both eyes he can he's seeing quite

60: $\quad$ good

61:NS $\mathrm{mm} \mathrm{hm}$

62:CR but if you cover his good eye he doesn't see the

63: television at all 
Again the caregiver interjects on line 56 to provide a different take on the problem from the patient's statement on line 54. In fact the patient's 'oh yes' on line 54 is ambiguous, as the nurse's question is not a Yes-No question - it could either be a positive response to being able to see the temperatures on TV or confirming that he has to get up close. However, the caregiver's interjection on Line 56 suggests that the 'oh yes' is a positive response, which she modifies by pointing out it is only because he is using his 'good eye'. The Nurse acknowledges 'right' and then affirms this with 'yeah', before stating that she would 'give you bit more on the score for that one'. She then proceeds with further questioning:

64:NS is there anything you've had to stop because of

65: $\quad$ your eyesight anything that you've had to stop

66: doing [that you like] doing nope

67:PT [no:::: ]

68:CR he has trouble he has trouble putting his glass

69: down doesn't he when he's got his drink

The patient continues to deny that his vision problems impair his daily activities on line 67 while the caregiver provides an alternative account on lines 68-69. The nurse's affiliative responses in this interactional sequence are borne out by her entries on the assessment sheet, where under the section on 'severity of visual impairment' she has scored the highest possible score 'severe' for all categories e.g. Do you have difficulty 'recognising people in the street', 'reading subtitles on TV', 'With leisure activities'. Similarly, under the section 'Ability to work, give care, live independently' a question asks 'Because of your vision, do you have difficulty caring for yourself'. The nurse has again circled the highest score 'severe' here. This is based on the following:

70:NS how about when you're doing things like (.) mm like

71: $\quad$ shaving and stuff

72:WF eh

73:NS can you see in the mirror alright(.)

74:PT ye[::s ]

75:NS [can] you see that you're not missing any bits

76:CR ((FIRST NAME- WF)) shaves him

77:NS yep

78:CR and he gets showered by the nurses

79:NS so the ( )

80:WF I think he does it by himself after that

81:NS does he [after the] beginning

82:WF [yeah ]

83:WF yeah he [done it] himself

$84: \mathrm{CR}$

$85: \mathrm{CR}$

[you ]

86:

87:WF

88:CR

89:NS

you but you finish it off you do all the

[( $)]$

90:WF I start it he finishes it 
The nurse then adds the score

91: $\quad$ so that's heaps 'two then two so that's nine ten

92: $\quad$ eleven (.) twenty nine (then one two $)^{\circ}$ three right

93: $\quad$ excellent (1.0) so what's wha-

94:CR does he qualify

95:NS yep you do

The nurse here has foreshadowed a good news outcome with 'excellent' on line 93, but the caregiver seeks clarification on line 94 with 'does he qualify' - perhaps suggesting that the word 'excellent' is not clearly linked to a particular activity by the nurse. Thus, the news in this case is delivered in a more perfunctory fashion than in the previous examples.

Later, the specialist enters the room again:

96:NS [thirty three ]

97:SP [how was the ] score yay

The nurse provides the score in overlap with the specialist's question followed by the specialist saying 'yay'.

We can see by the way in which the two consultations are organised the different types of organisational work being undertaken. Even with the separation of tasks there are difficulties posed for both the specialist and the nurse. The specialist is orienting to an operation, but still has to pose the (theoretical) possibility that the assessment will not reach the required points. The nurse then has to score, but her opening statement suggests this is a cursory matter that does not need close attention. The scoring then proceeds with the nurse appearing to attend to the information that will allow a classification of the highest scoring category. As such, she does not attend to the patient's statements which do not match, but to the caregiver's which do.

If the work of determining that an operation is needed is already done, the task now is to get alignment between that decision, the 'daily life' story of the patient and the assessment form. This is a separate task, where the production of the prioritisation score becomes an accomplishment that overrides any factors that might jeopardise the outcome already determined as appropriate by the specialist in the preceding consultation.

\section{Conclusion}

In the 1990s Freidson argued that:

The health-care system of today is best made sense of as a mix of the bureaucratic and professional models, with elements of the former rapidly growing in importance as the administrative structure surrounding practice expands (1994: 192).

The bureaucratic model overcomes the problem of a lack of trust in health professionals by laying down formal rules of conduct, but in so doing 'it undermines the flexible discretionary judgement that is necessary to adapt services to individual needs' (Freidson 1994:193). The three illustrative analyses of surgical consultations provided in this paper demonstrate that the complex work of the doctor-patient consultation may not necessarily be 'rational' in terms of a purely linear bureaucratic logic, but that it is in fact a highly professional 
'competent' and patient-centred activity. The challenge is to evolve decision-making tools or systems that not only acknowledge, but enhance, the interactional and intersubjective work that occurs in clinical interactions.

The nursing example bears out the findings of other research which shows that taskfocused formulaic interactions constrain nurses' usual responsiveness to patients. For example, in their research on nurses using a diabetes review checklist, Rhodes et al. (2006) argued that the clinical agenda of the checklist consistently superseded the patient's agenda. In addition the checklist led to interactional difficulties, where questions were asked out of context, and deviation from the checklist was discouraged. The focus on completing a certain task in a pre-defined routinised sequence has also been shown to make nurses less responsive to the topics and concerns raised by patients in other contexts, particularly where institutional documentation is involved, and the way forms are typically designed often encourages 'pursuit of a particular (type) of response' (Collins et al. 1997: 150).

We would suggest that it is therefore not unreasonable to suppose, by analogy, that the prioritisation agenda of the CPAC tools might well supersede the treatment plan agenda of the surgeons, and that surgeons would avoid this by using the tools post hoc outside the consultation. As we saw here, this means that when the nurse fills out the assessment form it is made to conform to the surgeon's agenda that has previously been agreed with the patient and not to the patient's particular responses to the questions asked.

According to Drew and Heritage (2006) 'CA focuses especially on those features of talk which are salient to participants' analyses of one another's turns at talk, in the progressive unfolding of interactions' (2006: xxx). In this paper we have focused on an 'absence' in the interaction, namely the non-use of protocols, and thus the very topic of interest is not apparently salient in the talk. However, the comparison above of the interactional features of a nurse consultation, where a protocol was explicitly used with those of the surgeons where it was not, lends credence to the argument made here that 'constructing a CPAC score' and 'determining a treatment plan' are distinctly different interactional activities. Moreover, there is clear evidence within the interactions of surgeons orienting to a protocol, even though its actual use is absent. Thus, in the second case, there is explicit reference to completing 'paperwork', referencing the requirements for booking a patient for surgery which include a CPAC score. In the third case, the registrar raises possible doubts about whether the proposed operation will proceed, referencing the fact that the nurse will subsequently be responsible for calculating the prerequisite CPAC score

In conclusion, a surgical consultation needs to be considered as more than simply the delivery of a service: it is an interactional accomplishment. The making of a diagnosis is an interactional as well as a clinical achievement, and the delivery of a diagnosis and a treatment plan is an interactionally complex matter that does not lend itself to the rigid following of a protocol. As Sharrock and Anderson (1986) argue in relation to the activities of scientists in laboratories, 'Any comic-strip conception of scientists as mechanical followers of an explicitly, general and invariable method of inquiry cannot withstand the simple evidence of the way in which inquiries are as much worked-up-as-they-go-along as they are done according to rigid, laid down formulae' (1986: 91). We would suggest that the same can be said for the process of determining whether surgery will or should be undertaken. Understanding the interaction can thus be seen as a delicate process of breaking good or bad news, and as a 'process' whose outcome is not totally predetermined but cannot be determined by following a formula.

It has been argued here that viewing diagnoses and treatment plans from the perspective of news delivery sequences provides insights into why protocols are not used in the consultation in the acquired data set. As Heath (1992) has noted, in the general-practice consultation turn design in the delivery of diagnosis and medical assessment facilitates patient cooperation and 
commitment to professional understandings. In the extracts analysed here it is clear that turn design is doing the same work in the surgeon consultation. Not only do the surgeon and patient co-operate in determining the outcome of the consultation, but at times the surgeon also undertakes careful management of the consultation in order that the patient will successfully align with professional understandings. The blunt instrument of a prioritisation tool does not allow for such intricate manoeuvres in relation to turn design.

It should also be acknowledged that diagnosis and planning care constitute different interactional activities to prioritisation on a waiting list. The implicit 'rules' that allow these activities to be identified as part of the consultation conflict with the explicit, externally imposed rules of administering a protocol that determines whether someone can access a rationed health service like elective surgery. Our data suggest that making and delivering a decision about operative thresholds is a non-linear interactional accomplishment that cannot easily be combined with the procedures presupposed by CPAC tools where a doctor would systematically work through symptoms and their impact on the patient to arrive at a score, which in turn decides priority for any treatment.

Although this research is based on a relatively small number of consultations, the analysis sheds light on important consultation processes that need to be more carefully considered when designing interventions to influence clinician behaviour. As Heath (1992) states 'It is critical that our practical recommendations to 'modify' the behaviour of medical practitioners...is thoroughly embedded in a detailed understanding of the local interactional organization and the various rights and responsibilities therein' (1992: 264). The challenge posed by this research is for policy makers and researchers who focus on health care interactions to develop appropriate 'tools' to enhance both equity and efficiency in health care delivery.

\section{Appendix}

(.) denotes a micro-pause

(2) denotes a pause of the specified number of seconds

(and) words in single parentheses denote candidate hearings

() parentheses without words denote indecipherable talk

((laughs)) double parentheses denote descriptions of action

[ ] square brackets denote overlapping talk.

$=\quad$ denotes latching or no gap between talk

$\underline{\text { surely }}$ underlined word denotes increased emphasis

denotes talk that is quieter that surrounding talk

:: $\quad$ colons denote prolongation of the immediately prior sound

Address for correspondence: Kevin Dew, Sociology Programme, Victoria University of Wellington, PO Box 600, Wellington 6140, New Zealand

e-mail: Kevin.dew@vuw.ac.nz

\section{Acknowledgements}

This research was funded by the New Zealand Health Research Council and the Marsden Fund. The research team would like to thank the participants for generously contributing their data, Dr Deborah McLeod and Rachel Tester for their input, and Sarah White for her 
part in the data collection. The research team would also like to thank the anonymous reviewers for their helpful comments.

\section{Notes}

1 Conversation analysts have shown that utterances are designed to invite a structurally 'preferred' or expected response - when a different response occurs, it is labelled as 'dispreferred' (Maynard 1992).

2 For the purposes of this paper the line numbers for each case are numbered consecutively; however, there are parts of the transcript not included in this paper.

\section{References}

Berg, M. (1997) Rationalizing Medical Work: Decision-support Techniques and Medical Practices, Cambridge: MIT Press.

Birkmeyer, J.D. and Welch, H.G. (1993) Rationing surgery: rules or constraints? Surgery, 113, 5, 491-7.

Collins, S., Britten, N., Ruusuvuori, J. and Thompson, A. (1997) Patient Participation in Health Care Consultations: Qualitative Perspectives. Berkshire: Open University Press/McGraw Hill Education.

Dew, K., Cumming, J., McLeod, D., Morgan, S., McKinlay, E., Dowell, A. and Love, T. (2005) Explicit rationing of elective services: implementing the New Zealand reforms, Health Policy, 74, $1,1-12$.

Diaz, F. (2000) The social organisation of chemotherapy treatment consultations, Sociology of Health and Illness, 22, 3, 364-89.

Drew, P. and Heritage, J. (2006) Editors' introduction, In Drew, P. and Heritage, J. (eds) Conversation Analysis:, Turn-taking and Repair, Volume 1. London: Sage.

Flynn, A. and Sinclair, M. (2005) Exploring the relationship between nursing protocols and nursing practice in an Irish intensive care unit, International Journal of Nursing Practice, 11, 4, $142-9$.

Freidson, E. (1994) Professionalism Reborn: Theory, Prophecy and Policy. Chicago: The University of Chicago Press.

Genuis, S.J. (2005) The proliferation of clinical practice guidelines: professional development or medicine-by-numbers, Journal of the American Board of Family Practice, 18, 5, 419-25.

Heath, C. (1992) The delivery and reception of diagnosis in the general-practice consultation. In Drew, P. and Heritage, J. (eds) Talk at Work: Interaction in Institutional Settings. Cambridge: Cambridge University Press.

Heritage, J. (1984) A change-of-state token and aspects of its sequential placement. In Maxwell Atkinson, J. and Heritage, J. (eds) Structures of Social Action: Studies in Conversation Analysis. Cambridge: Cambridge University Press.

Heritage, J. and Stivers, T. (1999) Online commentary in acute medical visits: a method of shaping patient expectations, Social Science and Medicine, 49, 11, 1501-17.

Heritage, J. and Maynard, D.W. (2006) Communication in medical care: interaction between primary care physicians and patients. Cambridge: Cambridge University Press.

Hutchinson, P., Read, R. and Sharrock, W. (2008) There is no such thing as a social science: in defence of Peter Winch. Aldershot: Ashgate.

Joyce, P. (2001) Governmentality and risk: setting priorities in the new NHS, Sociology of Health and Illness, 23, 5, 594-614.

Maynard, D.W. (1992) On clinicians co-implicating recipients' perspective in the delivery of diagnostic news. In Drew, P. and Heritage, J. (eds) Talk at Work: Interaction in Institutional Settings. Cambridge: Cambridge University Press. 
Maynard, D.W. (2003) Bad News, Good News: Conversational Order in Everyday Talk and Clinical Settings. Chicago: University of Chicago Press.

Maynard, D.W. and Frankel, R. (2006) On diagnostic rationality: bad news, good news, and the symptom residue. In Heritage, J. and Maynard, D.W. (eds) Communication in Medical Care: Interaction between Primary Care Physicians and Patients. Cambridge: Cambridge University Press.

McLeod, D., Morgan, S., McKinlay, E., Dew, K., Cumming, J., Dowell, A. and Love, T. (2004) Use of, and attitudes to, clinical priority assessment criteria in elective surgery in New Zealand, Journal of Health Services Research and Policy, 9, 2, 91-9.

Ministry of Health (2007) Elective Services: Orthopaedic CPAC explanation. Wellington: Ministry of Health.

Rhodes, P., Langdon, M., Rowley, E., Wright, J. and Small, N. (2006) What does the use of a computerized checklist mean for patient-centred care? The example of a routine diabetes review, Qualitative Health Research, 16, 3, 353-76.

Seale, C., Chaplin, R., Lelliot, P. and Quirk, A. (2007) Antipsychotic medication, sedation and mental clouding: an observational study of psychiatric consultations, Social Science and Medicine, 65, 4, 698-711.

Sharrock, W. and Anderson, B. (1986) The Ethnomethodologists. Chichester: Ellis Horwood.

Steinbrook, R. (2008) Saying no isn't NICE - the travails of Britain's National Institute for Health and Clinical Excellence, New England Journal of Medicine, 359, 19, 1977-81.

Taylor, M.C. and Hadorn, D. (2002) Developing priority criteria for general surgery: results from the Western Canada Waiting List Project, Canadian Journal of Surgery, 45, 5, 328-9. 